\title{
In situ Environmental TEM and DFT Studies on the Highly Stable AuIr Bimetallic Catalyst
}

\author{
Chang Wan Han $^{1}$, Paulami Majumdar ${ }^{2}$, Ernesto E. Marinero ${ }^{1}$, Antonio Aguilar-Tapia ${ }^{3}$, Rodolfo Zanella ${ }^{3}$, \\ Jeffrey Greeley ${ }^{2}$, and Volkan Ortalan ${ }^{1}$ \\ 1. School of Materials Engineering, Purdue University, West Lafayette, IN, USA \\ 2. School of Chemical Engineering, Purdue University, West Lafayette, IN, USA \\ 3. Centro de Ciencias Aplicadas y Desarrollo Technologico, Universidad Nacional Autonoma de Mexico, \\ Mexico City, Mexico
}

Since the report by Haruta et al. in 1987 of high catalytic activity of supported nanocrystalline Au catalysts in $\mathrm{CO}$ oxidation at low temperature [1], Au catalysts have attracted significant amount of interest from researchers. Despite the remarkable activity of Au catalysts in various oxidation processes, a wide range of uses of $\mathrm{Au}$ catalysts in industry is limited due to the lack of stability against sintering [2,3]. In this regard, stabilization of supported Au nanoparticles (NPs) is of utmost importance in the field of $\mathrm{Au}$ catalysis. With the purpose of increasing the stability of Au catalysts, various methods for increasing the stability have been developed [4,5]. One of the successful methods for increasing the stability is through adding a second metal into Au NPs [6-9]. Specifically, R. Zanella et al. reported that AuIr bimetallic NPs on $\mathrm{TiO}_{2}$ support show enhanced stability and activity compared to $\mathrm{Au}$ on $\mathrm{TiO}_{2}$ [7]. Considering several reports of the improved catalytic activity as well as on the improved stability of supported Au-based bimetallic catalysts, it can be expected that Au catalysts for industrial applications will be comprise bimetallic NPs rather than pure Au. Therefore, the mechanism behind the increased stability of Au-based bimetallic catalysts needs to be clearly understood. Despite the importance of Aubased bimetallic catalysts, however, the in situ investigations of their dynamic behaviors during the "real" catalytic process have been limited.

In this study, we performed in situ environmental TEM and DFT calculations to investigate the highly stable anatase $\mathrm{TiO} 2$ supported AuIr bimetallic NPs, prepared by the deposition-precipitation by urea (DPU) method [6,7]. Particularly, as an effort to understand the activity as well as the increased stability of AuIr catalyst, the dynamic behaviors (i.e., morphological variation and/or surface reconstruction) of AuIr NPs during room temperature CO oxidation process were investigated using in situ E-TEM technique. In all the experiments performed, significant care was taken to avoid electron beam damage by using of low-dose irradiation to avoid structural transformation of the Au NPs [10]. A mixture of CO and $\mathrm{He}(\mathrm{ratio}=1: 1)$ was fed into the differentially pumped environmental cell of the FEI Titan E-TEM and the pressure was maintained at $\sim 0.55$ Torr. Figure 1 shows the morphological variation of AuIr NPs in the $\mathrm{CO}$ and He ambient at room temperature. Fig. 1(a) displays a facetted AuIr NP at the onset of the experiment. Structural reconstruction occurs during the reaction and after 9 minutes of reaction time the morphology as shown in Fig. 1(b) is that of a rounded NP. Considering the fact that low-coordinated Au atoms [11], locating on the corners and edges of the NPs are attributed to the catalytic activity, the morphological variation can be correlated with the measured temporal variation of catalytic activity of AuIr NPs in CO oxidation. We also performed a series of density functional theory (DFT) calculations for single crystal AuIr alloy slabs on the $\mathrm{TiO}_{2}$ surface [Fig. 2], as a first approximation, to understand the higher stability of $\mathrm{TiO}_{2}$ supported AuIr NPs, as compared to Au NP. At the vacuum/metal interface, Au atoms prefer to segregate to the surface, but at the $\mathrm{TiO}_{2}$ interface, oxygen reverses this trend and draws 
the more oxophilic Ir atoms to the interface. The segregated $\mathrm{Ir}$ atoms near the $\mathrm{TiO}_{2}$ surface leads to the increase in stability of AuIr on $\mathrm{TiO}_{2}$. The results of in situ E-TEM analysis and DFT calculations will be provided and the correlation between the morphological changes of AuIr NPs with the activity in CO oxidation. Moreover, the mechanism of the increased stability will be discussed in the aspect of the theoretically calculated adsorption energies of AuIr slabs on $\mathrm{TiO}_{2}$.

\section{References}

[1] M. Haruta et al, Chem. Lett. 4 (1987) p. 405-408.

[2] T. Choudhary and D. Goodman, Top. Catal. 21 (2002) p. 25-34.

[3] M. José-Yacaman et al, J. Phys. Chem. B. 109 (2005) p. 9703-9711.

[4] M. Kirchhoff et al, Nanotechnology. 16 (2005) S401-S408.

[5] S.H. Joo et al, Nat. Mater. 8 (2009) p. 126-131.

[6] X. Bokhimi et al, J. Phys. Chem. C. 114 (2010) p. 14101-14109.

[7] A. Gomez-Cortes et al, J. Phys. Chem. C. 113 (2009) p. 9710-9720.

[8] Y. Guan et al, J. Catal. 305 (2013) p. 135-145.

[9] X. Liu et al, Chem. Commun. (2008) p. 3187-3189.

[10] Y. Kuwauchi et al, Angew. Chemie. 51 (2012) p. 7729-7733.

[11] B.. Hvolbæk et al, Nano Today. 2 (2007) p. 14-18.
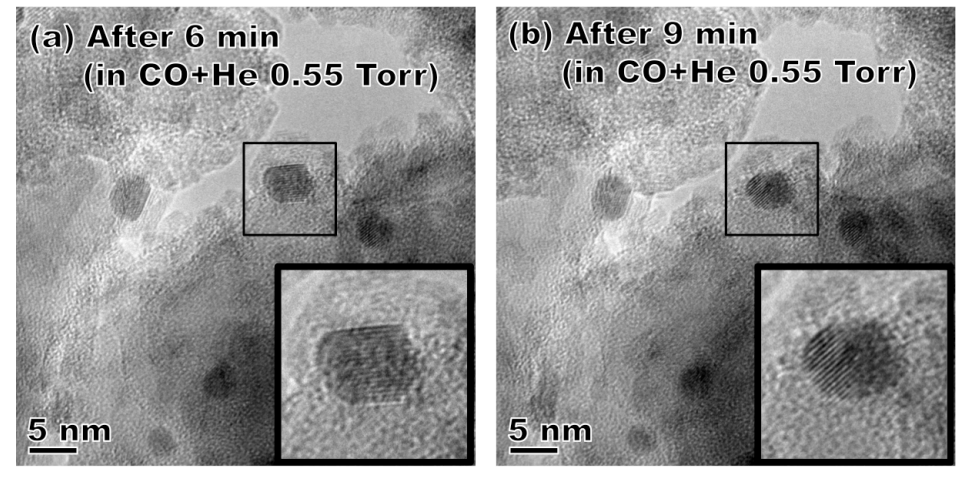

Figure 1. In situ E-TEM images of AuIr NPs on $\mathrm{TiO}_{2}$ after feeding the $\mathrm{CO}$ and $\mathrm{He}$ gas mixture. Morphological variation of a AuIr NP from the strongly facetted (Fig. 1a) to the rounded (Fig. 1b) is observed.

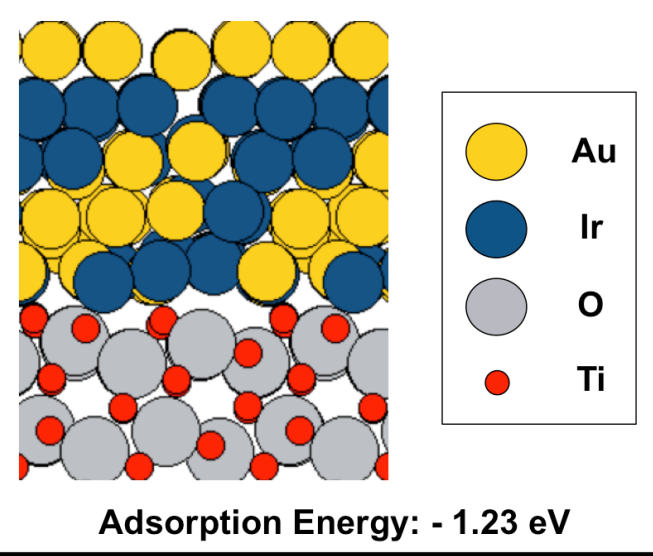

Figure 2. AuIr slab on anatase $\mathrm{TiO}_{2}$ surface showing segregation of $\mathrm{Au}$ to the vacuum interface and Ir to the support interface. Adsorption energy reported below figure. 